\title{
Canonical Banach function spaces generated by Urysohn universal spaces. Measures as Lipschitz maps
}

by

\author{
Piotr Niemiec (Kraków)
}

\begin{abstract}
It is proved (independently of the result of Holmes [Fund. Math. 140 (1992)]) that the dual space of the uniform closure $\operatorname{CFL}\left(\mathbb{U}_{r}\right)$ of the linear span of the maps $x \mapsto d(x, a)-d(x, b)$, where $d$ is the metric of the Urysohn space $\mathbb{U}_{r}$ of diameter $r$, is (isometrically if $r=+\infty$ ) isomorphic to the space $\operatorname{LIP}\left(\mathbb{U}_{r}\right)$ of equivalence classes of all real-valued Lipschitz maps on $\mathbb{U}_{r}$. The space of all signed (real-valued) Borel measures on $\mathbb{U}_{r}$ is isometrically embedded in the dual space of $\operatorname{CFL}\left(\mathbb{U}_{r}\right)$ and it is shown that the image of the embedding is a proper weak ${ }^{*}$ dense subspace of $\operatorname{CFL}\left(\mathbb{U}_{r}\right)^{*}$. Some special properties of the space $\operatorname{CFL}\left(\mathbb{U}_{r}\right)$ are established.
\end{abstract}

The unbounded Urysohn space was introduced in [13, 14]. Holmes [3] has proved that this space generates a unique (up to linear isometry) Banach space (for simpler proofs see [4], [8] or [10]). Such metric spaces are called linearly rigid. The Banach space generated by a linearly rigid metric

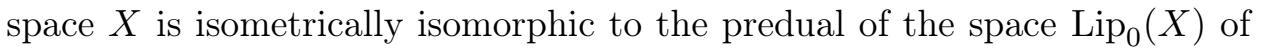
real-valued Lipschitz maps on $X$ vanishing at a fixed point of $X$ (equipped with the "Lipschitz" norm). It turns out that linearly rigid spaces are necessarily unbounded, provided they have more than two points (see [10]). This means that bounded Urysohn spaces do not generate unique Banach spaces. However, as we shall show, the dual space of some Banach function space generated by a bounded Urysohn space $\mathbb{U}_{r}$ is isomorphic to the space $\operatorname{Lip}_{0}\left(\mathbb{U}_{r}\right)$. The fundamental properties of Urysohn spaces will also enable us to link Borel measures with Lipschitz maps by means of a linear isometric map (given by a simple formula). However, the correspondence is not one-to-one, i.e. there are Lipschitz maps which do not "come from" measures.

Notation and terminology. The sets of all nonnegative reals and positive integers are denoted by $\mathbb{R}_{+}$and $\mathbb{N}_{*}$, respectively.

2000 Mathematics Subject Classification: 46E27, 26A16.

Key words and phrases: Urysohn's universal space, spaces of measures, spaces of Lipschitz maps. 
For a separable complete metric space $X, \operatorname{Mes}(X)$ stands for the Banach space of all signed (real-valued) Borel measures on $X$, equipped with the standard total variation norm. It is well known that each nonnegative (finite) Borel measure $\mu$ on $X$ is regular, i.e. $\mu(A)=\sup \{\mu(K) \mid K \subset A, K$ compact $\}$ for any Borel subset $A$ of $X$. This implies that the subspace $\operatorname{Mes}_{\mathrm{c}}(X)$ of $\operatorname{Mes}(X)$ consisting of all measures supported on compact sets is dense (with respect to the norm topology) in the whole space.

A Lipschitz map between metric spaces $(X, d)$ and $(Y, \varrho)$ is any function $f: X \rightarrow Y$ for which there is a finite constant $M \geq 0$ such that $\varrho(f(x), f(y)) \leq M d(x, y)$ for every $x, y \in X$. We denote by $\operatorname{Lip}(X)$ the space of all real-valued Lipschitz maps on $X$. If $X$ has more than one point, we equip the space $\operatorname{Lip}(X)$ with the following seminorm:

$$
l(f)=\sup _{\substack{x, y \in X \\ x \neq y}} \frac{|f(x)-f(y)|}{d(x, y)}, \quad f \in \operatorname{Lip}(X) .
$$

This is not a norm, because $l(f)=0$ if and only if $f$ is constant. Therefore we take the quotient space $\operatorname{Lip}(X) / \operatorname{Const}(X)$, where $\operatorname{Const}(X)$ consists of all (real-valued) constant maps on $X$, and denote it by $\operatorname{LIP}(X)$. The space $\operatorname{LIP}(X)$ is a Banach space with respect to its norm:

$$
L(f+\operatorname{Const}(X))=l(f), \quad f \in \operatorname{Lip}(X) .
$$

In what follows we shall write, for simplicity, $f \in \operatorname{LIP}(X)$ and $L(f)$ instead of $f \in \operatorname{Lip}(X)$ or $L(f+\operatorname{Const}(X))$. However, one has to remember that $\operatorname{LIP}(X)$ is not a function space. Nevertheless, if $x$ and $y$ are two points of $X$, the functional $\operatorname{LIP}(X) \ni f \mapsto f(x)-f(y) \in \mathbb{R}$ is well defined. Additionally, let $B_{L}(X)$ stand for the closed unit ball of $\operatorname{LIP}(X)$.

It is easy to see that $\operatorname{LIP}(X)$ is isometrically isomorphic to $\operatorname{Lip}_{0}(X, x)$, the subspace of $\operatorname{Lip}(X)$ constisting of the maps vanishing at $x$, where $x$ is any fixed point of $X$. Spaces of type $\operatorname{Lip}_{0}$ are well studied (see e.g. [15]). It is known that they are dual spaces, and the preduals are well described (the Arens-Eells spaces). For us, the two most important properties of $\operatorname{Lip}_{0}(X, x)$, after an adaptation to $\operatorname{LIP}(X)$, are (see also [11] for proofs):

(L1) If $X$ is separable, then the ball $B_{L}(X)$ is (compact and) metrizable in the weak ${ }^{*}$ topology, and a sequence $\left(f_{n}\right)_{n \in \mathbb{N}_{*}}$ of elements of $B_{L}(X)$ is weak* convergent to $f \in B_{L}(X)$ if and only if $f_{n}(x)-$ $f_{n}(y) \rightarrow f(x)-f(y)(n \rightarrow \infty)$ for all $x, y \in X$ (this condition, in fact, defines the weak* topology on $\left.B_{L}(X)\right)$.

(L2) Any weak* continuous functional $\psi: \operatorname{LIP}(X) \rightarrow \mathbb{R}$ has the form

$$
\psi(f)=\sum_{n=1}^{\infty} a_{n} \frac{f\left(x_{n}\right)-f\left(y_{n}\right)}{d\left(x_{n}, y_{n}\right)},
$$


where $\sum_{n=1}^{\infty}\left|a_{n}\right|<\infty$ and $\left(x_{n}\right)_{n \in \mathbb{N}_{*}}$ and $\left(y_{n}\right)_{n \in \mathbb{N}_{*}}$ are two sequences of elements of $X$ such that $x_{n} \neq y_{n}$; what is more, the sequences $\left(a_{n}\right)_{n \in \mathbb{N}_{*}},\left(x_{n}\right)_{n \in \mathbb{N}_{*}}$ and $\left(y_{n}\right)_{n \in \mathbb{N}_{*}}$ may be taken so that $\sum_{n=1}^{\infty}\left|a_{n}\right|$ is arbitrarily close to $\|\psi\|$.

Whenever we deal with spaces of real-valued maps, the symbol $\|\cdot\|$ denotes the supremum norm.

Now we pass to the main subject of the paper.

1. Definition. An Urysohn space is a separable complete metric space $X$ such that every separable metric space of diameter no greater than diam $X$ is isometrically embeddable in $X$, and each isometry between finite subsets of $X$ is extendable to an isometry of $X$ onto itself. An Urysohn space is nontrivial if it has more than one point.

For every $r \in[0,+\infty]$ there is a unique (up to isometry) Urysohn space of diameter $r$. We shall denote it by $\mathbb{U}_{r}$, and $\mathbb{U}$ will stand for the unbounded Urysohn space.

A Katětov map on a metric space $(X, d)$ is any function $f: X \rightarrow \mathbb{R}_{+}$ such that

$$
|f(x)-f(y)| \leq d(x, y) \leq f(x)+f(y) \quad \text { for all } x, y \in X .
$$

A common sphere is a set of the form

$$
S_{X}(A, f)=\{x \in X \mid \forall a \in A: d(x, a)=f(a)\}
$$

with nonempty $A \subset X$ and any function $f: A \rightarrow \mathbb{R}_{+}$. If $X$ is Urysohn, then $S_{X}(K, f)$ is nonempty for each nonempty compact subset $K$ of $X$ and every Katětov map $f$ on $K$ such that $f(K) \subset[0, \operatorname{diam} X]$. This is a consequence of the Huhunaišvili theorem [5]. For $r \in[0,+\infty]$, we denote by $E_{r}(X)$ the set of Katětov maps $f$ on $X$ such that $f(X) \subset[0, r]$. For more on Katětov maps, see [6], [8], [1]. The reader interested in Urysohn spaces is referred to $[7,8]$.

From now on, $r \in(0,+\infty], d$ is the metric of $\mathbb{U}_{r}$, and $B_{L}=B_{L}\left(\mathbb{U}_{r}\right)$. Let $\varrho: \mathbb{U}_{r} \rightarrow \operatorname{LIP}\left(\mathbb{U}_{r}\right)$ be defined as follows: $\varrho(x)$ is the equivalence class of the map $e_{x}$, where $e_{x}(y)=d(x, y)$. It is easy to see that $\varrho\left(\mathbb{U}_{r}\right) \subset B_{L}$, and $\varrho$ is continuous when $\operatorname{LIP}\left(\mathbb{U}_{r}\right)$ is considered with the weak* topology.

First we shall establish the basic properties of the set $\varrho\left(\mathbb{U}_{r}\right)$.

2. Proposition. The set $\varrho\left(\mathbb{U}_{r}\right)$ is linearly independent.

Proof. Let $n \geq 2$. Suppose that $x_{1}, \ldots, x_{n}$ are distinct points of $\mathbb{U}_{r}$ and $\alpha_{1}, \ldots, \alpha_{n}$ are scalars such that the map $u=\alpha_{1} e_{x_{1}}+\cdots+\alpha_{n} e_{x_{n}}$ is constant. Let $M=\operatorname{diam}\left\{x_{1}, \ldots, x_{n}\right\}$. For $j \in\{1, \ldots, n\}$, put $p_{j}=\min _{k \neq j} d\left(x_{j}, x_{k}\right)>0$. Let $A=\left\{x_{1}, \ldots, x_{n}\right\}$, let $f_{0}: A \rightarrow \mathbb{R}_{+}$be the constant map with value $M$, and for $j=1, \ldots, n$, let $f_{j}: A \rightarrow \mathbb{R}_{+}$be defined as follows: $f_{j}\left(x_{j}\right)=$ $M-p_{j}$ and $f_{j}\left(x_{k}\right)=M$ for $k \neq j$. It is easy to verify that $f_{0}, \ldots, f_{n}$ are 
Katětov maps and take values in $[0, r]$. There are points $z_{0}, \ldots, z_{n}$ such that $e_{x_{j}}\left(z_{k}\right)=f_{k}\left(x_{j}\right)$. Since the map $u$ is constant it follows that $u\left(z_{j}\right)=u\left(z_{0}\right)$, or equivalently $\sum_{m=1}^{n} \alpha_{m}\left(f_{j}\left(x_{m}\right)-f_{0}\left(x_{m}\right)\right)=0$. But this yields $\alpha_{j} p_{j}=0$ and thus $\alpha_{1}=\cdots=\alpha_{n}=0$.

3. TheOrem. The weak ${ }^{*}$ closure of $\varrho\left(\mathbb{U}_{r}\right)$ contains the ball $\frac{1}{2} B_{L}$. If $r=$ $+\infty$, then $\varrho\left(\mathbb{U}_{r}\right)$ is weak* dense in $B_{L}$.

Proof. First assume that $r=+\infty$. It is enough to show that for each $f \in B_{L}$ and any finite nonempty subset $A$ of $\mathbb{U}$ there are $C \in \mathbb{R}$ and $x \in \mathbb{U}$ such that $f+C=e_{x}$ on $A$. Since $A$ is finite, there is $C$ such that $d(a, b)-$ $f(a)-f(b) \leq 2 C$ for $a, b \in A$. This implies that $f+C$ is a Katětov map on $A$. So, there exists $x \in \mathbb{U}$ for which $f(a)+C=d(x, a)(a \in A)$. But this means that $f+C=e_{x}$ on $A$.

Now assume that $r$ is finite. Take $f \in B_{L}$ and a finite nonempty subset $A$ of $\mathbb{U}_{r}$. We have to prove that there are $C \in \mathbb{R}$ and $x \in \mathbb{U}_{r}$ such that $\frac{1}{2} f+C=e_{x}$ on $A$. Observe that since $L(f) \leq 1$ and diam $\mathbb{U}_{r}=r$, there is a constant $\alpha$ such that the image of $f+\alpha$ is contained in $\left[-\frac{1}{2} r, \frac{1}{2} r\right]$. But then the image of $\frac{1}{2} f+C$, where $C=\frac{1}{2} \alpha+\frac{3}{4} r$, is a subset of $\left[\frac{1}{2} r, r\right]$ and thus $f+C$ is a Katětov map on $A$ (because $\frac{1}{2} r \geq \frac{1}{2} \operatorname{diam} A$ ). So, as in the first case, it suffices to take $x \in \mathbb{U}_{r}$ such that $\frac{1}{2} f+C=e_{x}$ on $A$.

4. Corollary. If $\psi \in \operatorname{LIP}\left(\mathbb{U}_{r}\right)^{*}$ is weak $k^{*}$ continuous, then

$$
\frac{1}{2}\|\psi\| \leq \sup _{x \in \mathbb{U}_{r}} \psi(\varrho(x)) \leq\|\psi\|,
$$

and if $r=+\infty$, then $\|\psi\|=\sup _{x \in \mathbb{U}_{r}} \psi(\varrho(x))$.

5. Corollary. The set $\varrho(\mathbb{U})$ is metrizable in the weak* topology, but is not completely metrizable. In particular, $\varrho: \mathbb{U} \rightarrow \varrho(\mathbb{U})$ is not a homeomorphism.

Proof. Suppose that, on the contrary, $\varrho(\mathbb{U})$ is completely metrizable. Then, by Theorem 3 , it is a dense $\mathcal{G}_{\delta}$-subset of $B_{L}$ and thus so is $-\varrho(\mathbb{U})$. But $\varrho(\mathbb{U})$ and $-\varrho(\mathbb{U})$ are disjoint (thanks to Proposition 2), contrary to the Baire theorem.

Corollary 4 leads us to the following

6. Definition. The canonical function linear space (for short, the CFL space) of the Urysohn space $\mathbb{U}_{r}$ is the space $\mathrm{CFL}\left(\mathbb{U}_{r}\right)$ consisting of the maps $f: \mathbb{U}_{r} \rightarrow \mathbb{R}$ of type $f(x)=\psi(\varrho(x))$, where $\psi$ is a weak* continuous functional on $\operatorname{LIP}\left(\mathbb{U}_{r}\right)$. Since $\varrho\left(\mathbb{U}_{r}\right)$ is a subset of $B_{L}, \operatorname{CFL}\left(\mathbb{U}_{r}\right)$ consists of bounded maps. The CFL space is equipped with the supremum norm.

As an immediate consequence of Corollary 4 we obtain 
7. Theorem. The CFL space of the [unbounded] Urysohn space $\mathbb{U}_{r}$ is [isometrically] isomorphic to the predual of $\operatorname{LIP}\left(\mathbb{U}_{r}\right)$ and therefore it is a Banach space. The canonical [isometric] isomorphism $J: \operatorname{LIP}\left(\mathbb{U}_{r}\right)_{*} \rightarrow \operatorname{CFL}\left(\mathbb{U}_{r}\right)$ has the form

$$
(J(\psi))(x)=\psi(\varrho(x)) \quad\left(\psi \in \operatorname{LIP}\left(\mathbb{U}_{r}\right)_{*}, x \in \mathbb{U}_{r}\right),
$$

and $\max \left(\|J\|,\left\|J^{-1}\right\|\right) \leq 2$.

Now we will characterize the maps belonging to $\operatorname{CFL}\left(\mathbb{U}_{r}\right)$.

8. TheOREM. A function $f: \mathbb{U}_{r} \rightarrow \mathbb{R}$ is a member of $\mathrm{CFL}\left(\mathbb{U}_{r}\right)$ if and only if for any $\varepsilon>0$ there are $u_{1}, \ldots, u_{m} \in \mathbb{U}_{r}$ and $\alpha_{1}, \ldots, \alpha_{m} \in \mathbb{R}$ such that $\sum_{j=1}^{m} \alpha_{j}=0$ and for each $x \in \mathbb{U}_{r}$,

$$
\left|f(x)-\sum_{j=1}^{m} \alpha_{j} d\left(u_{j}, x\right)\right| \leq \varepsilon .
$$

Proof. If $f \in \operatorname{CFL}\left(\mathbb{U}_{r}\right)$, then, by (L2), there are sequences $\left(a_{n}\right)_{n=1}^{\infty} \in l^{1}$ and $\left(x_{n}, y_{n}\right)_{n=1}^{\infty} \in\left(\mathbb{U}_{r} \times \mathbb{U}_{r}\right)^{\mathbb{N}_{*}}$ such that $x_{n} \neq y_{n}$ and

$$
f(x)=\sum_{n=1}^{\infty} a_{n} \frac{d\left(x_{n}, x\right)-d\left(y_{n}, x\right)}{d\left(x_{n}, y_{n}\right)} .
$$

So, it is enough to take $N \geq 1$ such that $\sum_{n=N+1}^{\infty}\left|a_{n}\right|<\varepsilon$ and to express the map $x \mapsto \sum_{n=1}^{N} a_{n}\left(d\left(x_{n}, x\right)-d\left(y_{n}, x\right)\right) / d\left(x_{n}, y_{n}\right)$ in the form $\sum_{j=1}^{m} \alpha_{j} e_{u_{j}}$.

Now assume that $f$ is the uniform limit of a sequence of maps of the form $\sum_{j=1}^{m} \alpha_{j} e_{u_{j}}$ with $\sum_{j=1}^{m} \alpha_{j}=0$. It is easy to check that

$$
\left(\sum_{j=1}^{m} \alpha_{j} e_{u_{j}}\right)(x)=\psi(\varrho(x)),
$$

where $\psi: \operatorname{LIP}\left(\mathbb{U}_{r}\right) \rightarrow \mathbb{R}$ is a weak* continuous functional given by $\psi(g)=$ $\sum_{j=1}^{m} \alpha_{j} g\left(u_{j}\right)\left(g \in \operatorname{LIP}\left(\mathbb{U}_{r}\right)\right)$. So, $\sum_{j=1}^{m} \alpha_{j} e_{u_{j}} \in \operatorname{CFL}\left(\mathbb{U}_{r}\right)$ and thus the completeness of $\mathrm{CFL}\left(\mathbb{U}_{r}\right)$ finishes the proof.

9. Corollary. Let $f$ be a nonzero member of the CFL space of $\mathbb{U}$. Then the image $R$ of $f$ is a bounded interval such that $(-\|f\|,\|f\|) \subset R \subset$ $[-\|f\|,\|f\|]$.

Proof. By Theorem $8, f$ is continuous and thus $R$ is an interval. Further, thanks to Corollary $4,\|f\|$ belongs to the closure of $R$ and, similarly, $\|-f\|$ is in the closure of $-R$. This implies that the closure of $R$ coincides with $[-\|f\|,\|f\|]$. Now the assertion is clear.

In contrast to the case of the unbounded Urysohn space, for $r<+\infty$ the set $\varrho\left(\mathbb{U}_{r}\right)$ is not weak* dense in $B_{L}$. What is more, the canonical isomorphism $J: \operatorname{LIP}\left(\mathbb{U}_{r}\right)_{*} \rightarrow \operatorname{CFL}\left(\mathbb{U}_{r}\right)$ is nonisometric, as shown by 
10. Proposition. For $r<+\infty$, the convex hull $V$ of the set $\varrho\left(\mathbb{U}_{r}\right) \cup$ $\left(-\varrho\left(\mathbb{U}_{r}\right)\right)$ is not weak $k^{*}$ dense in $B_{L}$. In particular, the canonical isomorphism $J$ is nonisometric.

Proof. Take four points $p_{1}, p_{2}, p_{3}, p_{4}$ of $\mathbb{U}_{r}$ such that $d\left(p_{j}, p_{k}\right)=r$ for distinct $j, k \in\{1,2,3,4\}$. Let $g \in B_{L}$ be any nonexpansive map such that $g\left(p_{j}\right)=0$ and $g\left(p_{k}\right)=r$ for $j=1,2$ and $k=3$, 4. We claim that $g$ does not belong to the weak* closure $W$ of $V$. Suppose, for contradiction, that $g \in W$. This implies that there are numbers $t_{1}, \ldots, t_{n}$ and points $x_{1}, \ldots, x_{n}$ of $\mathbb{U}_{r}$ such that $\sum_{j=1}^{n}\left|t_{j}\right|=1$ and the map $g-\sum_{j=1}^{n} t_{j} e_{x_{j}}$ is constant on $A=\left\{p_{1}, p_{2}, p_{3}, p_{4}\right\}$. Since $e_{x} \in E_{r}\left(\mathbb{U}_{r}\right)$ for each $x \in \mathbb{U}_{r}$ and $E_{r}(A)$ is convex we infer that there is a $c \in \mathbb{R}$ such that

$$
\left.(g+c)\right|_{A} \in \operatorname{conv}\left[E_{r}(A) \cup\left(-E_{r}(A)\right)\right]
$$

("conv" stands for convex hull). It is easily seen that $\left.g\right|_{A}$, as an element of $\operatorname{LIP}(A)$, is an extreme point of $B_{L}(A)$. Thus, by (2), $\left.g\right|_{A}+c= \pm f$ for some $f \in E_{r}(A)$. This shows that $f\left(p_{1}\right)=f\left(p_{2}\right), f\left(p_{3}\right)=f\left(p_{4}\right)$ and $\mid f\left(p_{1}\right)-$ $f\left(p_{3}\right) \mid=r$. But $f(A) \subset[0, r]$ and therefore $f\left(p_{1}\right)=f\left(p_{2}\right)=0$ and $f\left(p_{3}\right)=$ $f\left(p_{4}\right)=r$ or conversely. So, card $f^{-1}(\{0\})>1$, which contradicts the relation $f \in E_{r}(A)$.

We have shown that $g \notin W$. By the separation theorem, there is a weak* continuous functional $\psi: \operatorname{LIP}\left(\mathbb{U}_{r}\right) \rightarrow \mathbb{R}$ such that $|\psi(u)| \leq 1$ for each $u \in W$ and $\psi(g)>1$. Hence $\|\psi\|>1$ and $\|J(\psi)\| \leq 1$, which finishes the proof.

Theorem 7 says that the dual of $\mathrm{CFL}\left(\mathbb{U}_{r}\right)$ may be identified with $\operatorname{LIP}\left(\mathbb{U}_{r}\right)$ (at least in the unbounded case). This identification has the following form: a functional $\varphi \in \mathrm{CFL}\left(\mathbb{U}_{r}\right)^{*}$ corresponds to a map $g \in \operatorname{LIP}\left(\mathbb{U}_{r}\right)$ such that $g(x)-g(y)=\psi\left(e_{x}-e_{y}\right)\left(x, y \in \mathbb{U}_{r}\right)$. For $f \in \operatorname{CFL}\left(\mathbb{U}_{r}\right)$ and $g \in \operatorname{LIP}\left(\mathbb{U}_{r}\right)$, we shall write $\int f \mathrm{~d} g$ or $\int f(x) \mathrm{d} g(x)$ for the value at $f$ of the functional corresponding to $g$. Thus for each $a, b \in \mathbb{U}_{r}$,

$$
\int\left(e_{a}-e_{b}\right) \mathrm{d} g=\int(d(a, x)-d(b, x)) \mathrm{d} g(x)=g(a)-g(b) .
$$

The next result will enable us to link measures with Lipschitz maps on Urysohn spaces.

11. TheOREM. Let $K$ be a (nonempty) compact subset of $\mathbb{U}_{r}$ and let $f: K \rightarrow \mathbb{R}$ be continuous. Then there is $F \in \operatorname{CFL}\left(\mathbb{U}_{r}\right)$ such that $\left.F\right|_{K}=f$ and $\|F\|=\|f\|$. What is more, for a given element $z$ of the common sphere $S_{\mathbb{U}_{r}}(K, s)$, where $s \in(0,+\infty)$ is such that $\frac{2}{3} \operatorname{diam} K \leq s \leq \frac{4}{5} r$, there are sequences $\left(x_{n}\right)_{n \in \mathbb{N}_{*}}$ and $\left(t_{n}\right)_{n \in \mathbb{N}_{*}}$ of elements of $\mathbb{U}_{r}$ and of positive numbers, respectively, such that $\|F\|=\sum_{n \in \mathbb{N}_{*}} t_{n} d\left(x_{n}, z\right)$ and $F=\sum_{n \in \mathbb{N}_{*}} t_{n}\left(e_{x_{n}}-e_{z}\right)$.

Proof. It is easily seen that $S_{\mathbb{U}_{r}}(K, s)$ is nonempty and $z \notin K$.

First assume that $f \in \operatorname{Lip}(K), l(f) \leq 1$ and $\|f\| \leq \frac{1}{4} s$. Define a map $g: K \cup\{z\} \rightarrow \mathbb{R}$ by $g(x)=f(x)+s$ for $x \in K$ and $g(z)=\|f\|$. The map $g$ 
is clearly nonexpansive on $K$ (i.e. $l\left(\left.g\right|_{K}\right) \leq 1$ ). What is more, for $x, y \in K$ we have $d(x, y) \leq \frac{3}{2} s \leq g(x)+g(y)$. Further,

$|g(x)-g(z)|=s-\|f\|+f(x) \leq s=d(x, z) \leq s+f(x)+\|f\|=g(x)+g(z)$.

So, $g$ is a Katětov map and $g(K \cup\{z\}) \subset[0, r]$. This implies that there is $u \in \mathbb{U}_{r}$ such that $g(y)=d(u, y)$ for $y \in K \cup\{z\}$. But then $f=\left.\left(e_{u}-e_{z}\right)\right|_{K}$ and $d(u, z)=g(z)=\|f\|$, and thus in that case the proof is finished.

Now consider an arbitrary map $f$. By [12], there are sequences $\left(t_{n}\right)_{n \in \mathbb{N}_{*}}$ and $\left(f_{n}\right)_{n \in \mathbb{N}_{*}}$ of positive numbers and real-valued nonexpansive maps on $K$ (respectively) such that $\|f\|=\sum_{n \in \mathbb{N}_{*}} t_{n}\left\|f_{n}\right\|$ and $f=\sum_{n \in \mathbb{N}_{*}} t_{n} f_{n}$. Replacing, if necessary, the pair $\left(t_{n}, f_{n}\right)$ by a suitable pair $\left(t_{n} / s_{n}, s_{n} f_{n}\right)$ with $s_{n} \in(0,1)$, we may assume that $\left\|f_{n}\right\| \leq \frac{1}{4} s$ for every $n$. We infer from the first part of the proof that there is a sequence $\left(x_{n}\right)_{n \in \mathbb{N}_{*}}$ of elements of $\mathbb{U}_{r}$ for which $\left.\left(e_{x_{n}}-e_{z}\right)\right|_{K}=f_{n}$ and $d\left(x_{n}, z\right)=\left\|f_{n}\right\|$. This implies that $\|f\|=\sum_{n \in \mathbb{N}_{*}} t_{n} d\left(x_{n}, z\right)$ and therefore the series $\sum_{n \in \mathbb{N}_{*}} t_{n}\left(e_{x_{n}}-e_{z}\right)$ is uniformly convergent. Let $F$ be its uniform limit. By Theorem $7, F \in \operatorname{CFL}\left(\mathbb{U}_{r}\right)$. Furthermore, $\left.F\right|_{K}=\sum_{n \in \mathbb{N}_{*}} t_{n} f_{n}=f$ and thus $\|f\| \leq\|F\|$. On the other hand, $\|F\| \leq \sum_{n \in \mathbb{N}_{*}} t_{n} d\left(x_{n}, z\right)=\|f\|$, which finishes the proof.

12. Corollary. Let $K$ be a (nonempty) compact subset of $\mathbb{U}_{r}$ and

$$
\Phi_{K}:\left.\operatorname{CFL}\left(\mathbb{U}_{r}\right) \ni f \mapsto f\right|_{K} \in \mathcal{C}(K),
$$

where $\mathcal{C}(K)$ is the algebra of all real-valued continuous functions on $K$. Then $\Phi_{K}$ sends the closed unit ball onto the closed unit ball and therefore the adjoint operator $\Phi_{K}^{*}: \operatorname{Mes}(K) \rightarrow \operatorname{LIP}\left(\mathbb{U}_{r}\right)$ is a weak ${ }^{*}$ continuous isomorphic (and isometric if $r=+\infty$ ) embedding such that $\max \left(\left\|\Phi_{K}^{*}\right\|,\left\|\left(\Phi_{K}^{*}\right)^{-1}\right\|\right) \leq 2$. If $\mu \in \operatorname{Mes}(K)$ and $g=\Phi_{K}^{*}(\mu)$, then for each $f \in \operatorname{CFL}\left(\mathbb{U}_{r}\right)$,

$$
\int_{K} f \mathrm{~d} \mu=\int f \mathrm{~d} g .
$$

In particular, $\Phi_{K}^{*}\left(\delta_{a}\right)=\varrho(a)$ for $a \in K$, where $\delta_{a}$ is the Dirac measure at a.

13. Lemma. Let $K$ and $L$ be two (nonempty) compact subsets of $\mathbb{U}_{r}$. If $\mu \in \operatorname{Mes}\left(\mathbb{U}_{r}\right)$ is a measure supported on $K \cap L$ (and therefore $\mu$ may be seen as a member of $\operatorname{Mes}(K)$ and $\operatorname{Mes}(L))$, then $\Phi_{K}^{*}(\mu)=\Phi_{L}^{*}(\mu)$.

Proof. Let $g=\Phi_{K}^{*}(\mu)$ and $h=\Phi_{L}^{*}(\mu)$. Then for any $f \in \operatorname{CFL}\left(\mathbb{U}_{r}\right)$ we have

$$
\int f \mathrm{~d} g=\int_{K} f \mathrm{~d} \mu=\int_{K \cap L} f \mathrm{~d} \mu=\int_{L} f \mathrm{~d} \mu=\int f \mathrm{~d} h,
$$

which implies that $g=h$.

The above lemma enables us to define an operator $j_{0}: \operatorname{Mes}_{c}\left(\mathbb{U}_{r}\right) \rightarrow$ $\operatorname{LIP}\left(\mathbb{U}_{r}\right)$ by $j_{0}(\mu)=\Phi_{K}^{*}(\mu)$, where $K$ is a compact subset of $\mathbb{U}_{r}$ such that $\mu$ is supported on $K$. Since the definition is independent of the choice of $K$, 
the map $j_{0}$ is a linear embedding such that $\max \left(\left\|j_{0}\right\|,\left\|j_{0}^{-1}\right\|\right) \leq 2$ and thus it is uniquely extendable to an isomorphic embedding of $\operatorname{Mes}\left(\mathbb{U}_{r}\right)$ in $\operatorname{LIP}\left(\mathbb{U}_{r}\right)$. We introduce the following

14. Definition. The canonical embedding of $\operatorname{Mes}\left(\mathbb{U}_{r}\right)$ in $\operatorname{LIP}\left(\mathbb{U}_{r}\right)$ is a unique continuous extension $j: \operatorname{Mes}\left(\mathbb{U}_{r}\right) \rightarrow \operatorname{LIP}\left(\mathbb{U}_{r}\right)$ of $j_{0}$. The canonical embedding is an isomorphism between its domain and range which sends Dirac's measure $\delta_{x}$ to $\varrho(x)$ for each $x \in \mathbb{U}_{r}$. What is more, the formula (4) is satisfied for any $\mu \in \operatorname{Mes}\left(\mathbb{U}_{r}\right)$ with $g=j(\mu)$ and $K$ replaced by $\mathbb{U}_{r}$. If $r=+\infty$, then $j$ is isometric.

The next result can be easily obtained from (4) by substituting $f=$ $e_{x}-e_{y}$.

15. Theorem. Let $\mu \in \operatorname{Mes}\left(\mathbb{U}_{r}\right)$ and $g=j(\mu)$. Then for each $x, y \in \mathbb{U}_{r}$,

$$
g(x)-g(y)=\int_{\mathbb{U}_{r}}(d(x, z)-d(y, z)) \mathrm{d} \mu(z) .
$$

It is rather surprising that $j$ is isometric also for bounded Urysohn spaces. We shall prove this in the following

16. Proposition. For $r<+\infty$, the canonical embedding $j: \operatorname{Mes}\left(\mathbb{U}_{r}\right) \rightarrow$ $\operatorname{LIP}\left(\mathbb{U}_{r}\right)$ is isometric.

Proof. Let $K$ be a compact nonempty subset of $\mathbb{U}_{r}$ and let $\mu \in \operatorname{Mes}\left(\mathbb{U}_{r}\right)$. Put $g=j(\mu)$. It is enough to check that $\|g\| \geq\|\mu\|-\varepsilon$ for $\varepsilon>0$. Since the space $\operatorname{Lip}(K)$ is dense in $\mathcal{C}(K)$, there is $u \in \operatorname{Lip}(K)$ such that $\|u\|=1$ and $\int_{K} u \mathrm{~d} \mu \geq\|\mu\|-\varepsilon$. Take $t>0$ such that $l(t u) \leq 1$ and $\|t u\| \leq \frac{3}{16} r$. It follows from the proof of Theorem 11 that there are $x, z \in \mathbb{U}_{r}$ for which $u=\left.\frac{1}{t}\left(e_{x}-e_{z}\right)\right|_{K}$. This yields $t=\left\|e_{x}-e_{z}\right\|=d(x, z)$ and thus

$$
\|\mu\|-\varepsilon \leq \int_{K} u \mathrm{~d} \mu=\int_{K} \frac{e_{x}-e_{z}}{d(x, z)} \mathrm{d} \mu=\frac{g(x)-g(z)}{d(x, z)} \leq\|g\| .
$$

17. Corollary. The norm closure of the linear span of $\varrho\left(\mathbb{U}_{r}\right)$ in $\operatorname{LIP}\left(\mathbb{U}_{r}\right)$ is (naturally) isometrically isomorphic to $l^{1}\left(\varrho\left(\mathbb{U}_{r}\right)\right)$.

18. Corollary. For any $\mu \in \operatorname{Mes}\left(\mathbb{U}_{r}\right)$, the total variation $|\mu|\left(\mathbb{U}_{r}\right)$ of the measure $\mu$ satisfies the condition

$$
|\mu|\left(\mathbb{U}_{r}\right)=\sup \left\{\left|\int_{\mathbb{U}_{r}} \frac{d(x, z)-d(y, z)}{d(x, y)} \mathrm{d} \mu(z)\right|: x, y \in \mathbb{U}_{r}, x \neq y\right\} .
$$

Our next aim is to prove that the canonical embedding $j$ is nonsurjective. We shall do this using different methods for bounded and unbounded Urysohn spaces.

It is folklore that $\varrho\left(\mathbb{U}_{r}\right)$ consists of extreme points of the ball $B_{L}$. However, $\varrho\left(\mathbb{U}_{r}\right) \cup\left(-\varrho\left(\mathbb{U}_{r}\right)\right)$ is a proper subset of the set of all extreme points 
of $B_{L}$, as we shall see below. In fact, for $r=+\infty$, this is a consequence of the following

19. Lemma. Let $(Z, \lambda)$ be a metric space and $A$ its nonempty subset. If $f \in \operatorname{LIP}(A)$ is an extreme point of $B_{L}(A)$, then the Katětov extension $\widehat{f}$ of $f$ is an extreme point of $B_{L}(Z)$, where $\widehat{f}(z)=\inf _{a \in A}(f(a)+\lambda(a, z))$.

Proof. It is easily checked that $\widehat{f} \in B_{L}(Z)$ and $\widehat{f}$ extends $f$. What is more, $\widehat{f}$ is the greatest element (with respect to the pointwise order) among nonexpansive extensions of $f$. So, if $g_{1}, g_{2} \in B_{L}(Z)$ are such that $\widehat{f}=\left(g_{1}+g_{2}\right) / 2+C$ for some constant $C$, then $f=\left(\left.g_{1}\right|_{A}+\left.g_{2}\right|_{A}\right) / 2+C$ and thus $f=\left.g_{1}\right|_{A}+C_{1}=\left.g_{2}\right|_{A}+C_{2}$, where $C_{1}$ and $C_{2}$ are constants with $C_{1}+C_{2}=2 C$. Thus $g_{j}+C_{j} \leq \widehat{f}(j=1,2)$. But $g_{1}+C_{1}+g_{2}+C_{2}=2 \widehat{f}$ and therefore $\widehat{f}=g_{1}+C_{1}=g_{2}+C_{2}$.

20. Proposition. There are extreme points of $B_{L}$ which do not belong to $\varrho(\mathbb{U}) \cup(-\varrho(\mathbb{U}))$. In particular, the canonical embedding $j: \operatorname{Mes}(\mathbb{U}) \rightarrow$ $\mathrm{LIP}(\mathbb{U})$ is not surjective.

Proof. Let $A$ be a subset of $\mathbb{U}$ which is isometric to $\mathbb{R}$ and let $\varphi: A \rightarrow \mathbb{R}$ be an isometry. Since the operator $\operatorname{LIP}(\mathbb{R}) \ni u \mapsto u \circ \varphi \in \operatorname{LIP}(A)$ is an isometric isomorphism and the map $f: \mathbb{R} \ni t \mapsto t \in \mathbb{R}$ is an extreme point of $B_{L}(\mathbb{R})$, it follows that $\varphi=f \circ \varphi$ is an extreme point of $B_{L}(A)$. So, by Lemma $19, v=\widehat{\varphi}$ is extreme in $B_{L}$. Observe that $v(\mathbb{U})=\mathbb{R}$, from which we infer that $v \notin \varrho(\mathbb{U}) \cup(-\varrho(\mathbb{U}))$. Finally, since the set $j^{-1}(\varrho(\mathbb{U}) \cup(-\varrho(\mathbb{U})))$ consists of all extreme points of the closed unit ball of $\operatorname{Mes}(\mathbb{U})$ (and $j$ is isometric), it follows that $v$ is not the value of $j$.

Now we have to show the same for a bounded Urysohn space. In order to do that, we need the following

21. Lemma. Let $r<+\infty$ and let $\left\{a_{n}: n \geq 1\right\}$ be a dense subset of $\mathbb{U}_{r}$. If $g \in \operatorname{LIP}\left(\mathbb{U}_{r}\right)$ is an element of the image of $j$, then for any $\varepsilon>0$ there exists $N \geq 1$ such that

$$
|g(x)-g(y)| \leq\|g\| \cdot\left\|\left.\left(e_{x}-e_{y}\right)\right|_{A}\right\|+\varepsilon
$$

for all $x, y \in \mathbb{U}_{r}$, where $A=\left\{a_{1}, \ldots, a_{N}\right\}$.

Proof. We may assume that $g \neq 0$. Let $\mu=j^{-1}(g)$. There is a compact nonempty subset $K$ of $\mathbb{U}_{r}$ such that $|\mu|\left(\mathbb{U}_{r} \backslash K\right) \leq \varepsilon / 3 r$. Since $K$ is compact, there are $x_{1}, \ldots, x_{p} \in K$ such that $K \subset \bigcup_{j=1}^{p} B\left(x_{j}, \varepsilon / 6\|g\|\right)$. Finally, there are positive integers $m_{1}, \ldots, m_{p}$ such that $d\left(x_{j}, a_{m_{j}}\right) \leq \varepsilon / 6\|g\|$ for $j=1, \ldots, p$. Put $N=\max \left(m_{1}, \ldots, m_{p}\right)$ and $A=\left\{a_{1}, \ldots, a_{N}\right\}$. Take $x, y \in \mathbb{U}_{r}$. By the triangle inequality, for each $z \in K$ there is $a \in A$ for which $|d(x, z)-d(y, z)| \leq|d(x, a)-d(y, a)|+2 \varepsilon / 3\|g\|$ and thus $\left\|\left.\left(e_{x}-e_{y}\right)\right|_{K}\right\| \leq$ $\left\|\left.\left(e_{x}-e_{y}\right)\right|_{A}\right\|+2 \varepsilon / 3\|g\|$. This yields 


$$
\begin{aligned}
|g(x)-g(y)| & =\left|\int_{\mathbb{U}_{r}}(d(x, z)-d(y, z)) \mathrm{d} \mu(z)\right| \\
& \leq \int_{K}\left|e_{x}-e_{y}\right| \mathrm{d}|\mu|+\int_{\mathbb{U}_{r} \backslash K}\left|e_{x}-e_{y}\right| \mathrm{d}|\mu| \\
& \leq\left(\left\|\left.\left(e_{x}-e_{y}\right)\right|_{A}\right\|+\frac{2 \varepsilon}{3\|g\|}\right)|\mu|\left(\mathbb{U}_{r}\right)+r \cdot|\mu|\left(\mathbb{U}_{r} \backslash K\right) \\
& \leq\|g\| \cdot\left\|\left.\left(e_{x}-e_{y}\right)\right|_{A}\right\|+\varepsilon .
\end{aligned}
$$

And now the announced result:

22. Proposition. For $r<+\infty$, the canonical embedding $j$ is nonsurjective. There are extreme points of $B_{L}$ which do not belong to $\varrho\left(\mathbb{U}_{r}\right) \cup\left(-\varrho\left(\mathbb{U}_{r}\right)\right)$.

Proof. Let $\left(U_{n}\right)_{n \geq 1}$ be a sequence of nonempty open subsets of $\mathbb{U}_{r}$ which form a basis of the topology of $\mathbb{U}_{r}$. Take any $x_{1} \in U_{1}$ and put $g\left(x_{1}\right)=0$. Now suppose that we have found points $x_{1}, \ldots, x_{3 k-2}$ of $\mathbb{U}_{r}$ and have defined $g\left(x_{1}\right), \ldots, g\left(x_{3 k-2}\right)$ (for some $k \geq 1$ ) in such a way that for any $j \in\{1, \ldots, k\}$ :

$(1)_{j}\left\{x_{1}, \ldots, x_{3 j-2}\right\} \cap U_{j} \neq \emptyset$,

$(2)_{j}\left|g\left(x_{p}\right)-g\left(x_{q}\right)\right| \leq d\left(x_{p}, x_{q}\right)$ and $g\left(x_{p}\right) \in[0, r]$ for $p, q=1, \ldots, 3 j-2$,

$(3)_{j}$ if $j>1$, then $\left|g\left(x_{3 j-4}\right)-g\left(x_{3 j-3}\right)\right|=r$ and $e_{x_{3 j-4}}=e_{x_{3 j-3}}$ on the set $\left\{x_{1}, \ldots, x_{3 j-5}\right\}$.

Take $x_{3 k-1}, x_{3 k} \in \mathbb{U}_{r}$ such that $d\left(x_{p}, x_{q}\right)=r$ for $p=1, \ldots, 3 k-2$ and $q=3 k-1,3 k$ and $d\left(x_{3 k-1}, x_{3 k}\right)=r$. Put $g\left(x_{3 k-1}\right)=r$ and $g\left(x_{3 k}\right)=0$. Now pick any $x_{3 k+1} \in U_{k+1} \backslash\left\{x_{1}, \ldots, x_{3 k}\right\}$ and define $g\left(x_{3 k+1}\right)=\min \left\{g\left(x_{j}\right)\right.$ $\left.+d\left(x_{j}, x_{3 k+1}\right): j \in\{1, \ldots, 3 k\}\right\}$. There is no difficulty in checking that $g\left(x_{3 k+1}\right) \in[0, r]$ and that the conditions $(1)_{k+1}-(3)_{k+1}$ hold. Thus we have obtained sequences $\left(x_{n}\right)_{n \in \mathbb{N}_{*}}$ and $\left(g\left(x_{n}\right)\right)_{n \in \mathbb{N}_{*}}$ such that the set $D=\left\{x_{n}\right.$ : $n \geq 1\}$ is dense in $\mathbb{U}_{r}$, the map $g: D \rightarrow \mathbb{R}$ is nonexpansive and for any finite subset $C$ of $D$ there are $x, y \in D$ such that $e_{x}=e_{y}$ on $C$ and $|g(x)-g(y)|=r$. Let $h \in B_{L}$ be the unique nonexpansive extension of $g$. The properties of $g$ and Lemma 21 imply that $h \notin j\left(\operatorname{Mes}\left(\mathbb{U}_{r}\right)\right)$.

Now suppose that the set of all extreme points of $B_{L}$ coincides with $M=\varrho\left(\mathbb{U}_{r}\right) \cup\left(-\varrho\left(\mathbb{U}_{r}\right)\right)$. As $B_{L}$ is metrizable in the weak ${ }^{*}$ topology, the Choquet theorem yields a Borel probability measure $\lambda$ on $M$ such that

$$
\int_{M} u \mathrm{~d} \lambda(u)=h .
$$

Further, since $\mathbb{U}_{r} \ni x \mapsto \varrho(x) \in \varrho\left(\mathbb{U}_{r}\right)$ is a continuous bijection, $\varrho\left(\mathbb{U}_{r}\right)$ is a Borel subset of $M$ and the inverse function is Borel. Let $\mu_{1}, \mu_{2} \in \operatorname{Mes}\left(\mathbb{U}_{r}\right)$ be defined by $\mu_{1}(A)=\lambda(\varrho(A))$ and $\mu_{2}(A)=\lambda(-\varrho(A))$ for a Borel subset $A$ of $\mathbb{U}_{r}$, and let $\mu=\mu_{1}-\mu_{2}$. Fix $x, y \in \mathbb{U}_{r}$. Since the functional $\operatorname{LIP}\left(\mathbb{U}_{r}\right) \ni$ 
$u \mapsto u(x)-u(y) \in \mathbb{R}$ is weak* continuous, by (7) and the measure transport theorem,

$$
\begin{aligned}
h(x)-h(y) & =\int_{M}(u(x)-u(y)) \mathrm{d} \lambda(u) \\
& =\int_{\varrho\left(\mathbb{U}_{r}\right)}(u(x)-u(y)) \mathrm{d} \lambda(u)+\int_{-\varrho\left(\mathbb{U}_{r}\right)}(u(x)-u(y)) \mathrm{d} \lambda(u) \\
& =\int_{\mathbb{U}_{r}}(\varrho(z)(x)-\varrho(z)(y)) \mathrm{d} \mu_{1}(z)+\int_{\mathbb{U}_{r}}(-\varrho(z)(x)+\varrho(z)(y)) \mathrm{d} \mu_{2}(z) \\
& =\int_{\mathbb{U}_{r}}(d(x, z)-d(y, z)) \mathrm{d} \mu(z),
\end{aligned}
$$

which means that $h=j(\mu)$. But this contradicts the first part of the proof.

23. REmark. The nonsurjectivity of $j$ in the case of a bounded Urysohn space may be immediately deduced from Propositions 10 and 16. Indeed, it is easy to check that if $\Psi: \operatorname{Mes}\left(\mathbb{U}_{r}\right) \rightarrow \operatorname{CFL}\left(\mathbb{U}_{r}\right)^{*}$ is an operator defined by $\Psi(\mu)(f)=\int_{\mathbb{U}_{r}} f \mathrm{~d} \mu$, then $\Psi$ is isometric (by Theorem 11 or Corollary 12) and $j=J \circ \Psi$. The same argument shows that $\left.J\right|_{E}$ is an isometry between $E=\Psi\left(\operatorname{Mes}\left(\mathbb{U}_{r}\right)\right)$ and $F=j\left(\operatorname{Mes}\left(\mathbb{U}_{r}\right)\right)$. What is more, $J$, as a dual operator, is a weak* homeomorphism and the spaces $E$ and $F$ are weak ${ }^{*}$ dense in $\operatorname{CFL}\left(\mathbb{U}_{r}\right)^{*}$ and $\operatorname{LIP}\left(\mathbb{U}_{r}\right)$, respectively. So, we have obtained an interesting example of a weak* homeomorphism which is isometric on a weak* dense subspace of the domain, but not isometric on the whole domain.

Our last aim is to establish some geometric properties of the space $\operatorname{CFL}\left(\mathbb{U}_{r}\right)$. The property (L2) implies that $B_{L}$ is the closed convex hull of the set $\mathrm{CFL}_{0}\left(\mathbb{U}_{r}\right)=\left\{\left(e_{x}-e_{y}\right) / d(x, y): x, y \in \mathbb{U}_{r}, x \neq y\right\}$. The next result shows that the set $\mathrm{CFL}_{0}(\mathbb{U})$ is transitive with respect to isometric isomorphisms of $\mathrm{CFL}(\mathbb{U})$.

24. TheOREM. For any $f, g \in \mathrm{CFL}_{0}(\mathbb{U})$ there exists an isometric isomorphism $V: \mathrm{CFL}(\mathbb{U}) \rightarrow \mathrm{CFL}(\mathbb{U})$ such that $V(f)=g$.

Proof. Let $(p, q)$ and $(a, b)$ be pairs of distinct points of $\mathbb{U}$ such that

$$
f=\frac{e_{p}-e_{q}}{d(p, q)} \quad \text { and } \quad g=\frac{e_{a}-e_{b}}{d(a, b)} .
$$

There is a bijection $\varphi: \mathbb{U} \rightarrow \mathbb{U}$ such that $\varphi(p)=a, \varphi(q)=b$ and $d(\varphi(x), \varphi(y))$ $=\lambda d(x, y)$ for any $x, y \in \mathbb{U}$, where $\lambda=d(a, b) / d(p, q)$. Now let $V: \operatorname{CFL}(\mathbb{U}) \rightarrow$ $\mathrm{CFL}(\mathbb{U})$ be the linear operator defined by $V(h)=h \circ \varphi^{-1}(h \in \mathrm{CFL}(\mathbb{U}))$. The map $V$ is well defined, because

$$
V\left(e_{x}-e_{y}\right)=\frac{e_{\varphi(x)}-e_{\varphi(y)}}{\lambda} .
$$

It is clearly a bijective isometric map and (8) shows that $V(f)=g$. 
Now let $\omega \in \mathbb{U}_{r}$ and $A_{\omega}=\left\{e_{x}-e_{\omega}: x \in \mathbb{U}_{r}\right\} \subset \operatorname{CFL}\left(\mathbb{U}_{r}\right)$. Note that $0 \in A_{\omega}$ and the map $m_{\omega}: \mathbb{U}_{r} \ni x \mapsto e_{x}-e_{\omega} \in A_{\omega}$ is isometric, so the set $A_{\omega}$ is closed. It is also a total subset of $\mathrm{CFL}\left(\mathbb{U}_{r}\right)$. First we shall prove the following

25. Proposition. The set $A_{\omega} \backslash\{0\}$ is linearly independent.

Proof. Let $x_{1}, \ldots, x_{n}$ be distinct elements of $\mathbb{U}_{r} \backslash\{\omega\}$ and $\alpha_{1}, \ldots, \alpha_{n}$ be real numbers such that $\sum_{j=1}^{n} \alpha_{j}\left(e_{x_{j}}-e_{\omega}\right)=0$. This implies that $\sum_{j=1}^{n} \alpha_{j} \varrho\left(x_{j}\right)$ $=\left(\sum_{j=1}^{n} \alpha_{j}\right) \varrho(\omega)$. So, Proposition 2 finishes the proof.

To state the next result, we need an auxiliary notion. For a number $\lambda \in(0,+\infty)$, we say that a function $w: P \rightarrow Q$ between metric spaces $(P, p)$ and $(Q, q)$ is $\lambda$-isometric if

$$
q(w(x), w(y))=\lambda p(x, y) \quad \text { for each } x, y \in P .
$$

Additionally, set $\Lambda(P)=\{1\}$ if $P$ is bounded, and $\Lambda(P)=(0,+\infty)$ otherwise. Now we are ready to present

26. Theorem. Let $\omega, \tau \in \mathbb{U}_{r}$. Let $K$ be a nonempty compact subset of $A_{\omega}$ and let $v: K \rightarrow A_{\tau}$ be $\lambda$-isometric with $\lambda \in \Lambda\left(\mathbb{U}_{r}\right)$. Then there is an isometric isomorphism $V: \operatorname{CFL}\left(\mathbb{U}_{r}\right) \rightarrow \mathrm{CFL}\left(\mathbb{U}_{r}\right)$ and $f_{0} \in A_{\tau}$ such that $v(f)=\lambda V(f)+f_{0}$ for every $f \in K$.

Proof. Let $K_{0}=m_{\omega}^{-1}(K)$ and $u: K_{0} \ni x \mapsto m_{\tau}^{-1}\left(v\left(m_{\omega}(x)\right)\right) \in \mathbb{U}_{r}$. The set $K_{0}$ is compact and $u$ is $\lambda$-isometric, so there is a bijective $\lambda$-isometric $\operatorname{map} U: \mathbb{U}_{r} \rightarrow \mathbb{U}_{r}$ which extends $u$. Now put $V: \operatorname{CFL}\left(\mathbb{U}_{r}\right) \ni f \mapsto f \circ U^{-1} \in$ $\operatorname{CFL}\left(\mathbb{U}_{r}\right)$ and $f_{0}=e_{U(\omega)}-e_{\tau} \in A_{\tau}$. As in the proof of Theorem 24, $V$ is an isometric isomorphism such that $V\left(e_{x}-e_{y}\right)=\left(e_{U(x)}-e_{U(y)}\right) / \lambda$. So, if $x \in \mathbb{U}_{r}$ and $f=e_{x}-e_{\omega}$, then

$$
\begin{aligned}
v(f) & =v\left(m_{\omega}(x)\right)=m_{\tau}(u(x))=m_{\tau}(U(x))=e_{U(x)}-e_{\tau} \\
& =\lambda V\left(e_{x}-e_{\omega}\right)+e_{U(\omega)}-e_{\tau}=\lambda V(f)+f_{0} .
\end{aligned}
$$

27. REMARK. As mentioned at the beginning of the paper, the fact that the dual of $\mathrm{CFL}(\mathbb{U})$ is linearly isometric to $\operatorname{LIP}(\mathbb{U})$ is a consequence of the Holmes theorem $[3,4]$. Namely, he has shown that if $(E,\|\cdot\|)$ is any Banach space such that $\mathbb{U} \subset E$ and $\|x-y\|=d(x, y)$ for all $x, y \in \mathbb{U}$, then for any $x_{1}, \ldots, x_{n} \in \mathbb{U}$ and $\alpha_{1}, \ldots, \alpha_{n} \in \mathbb{R}$ with $\sum_{j=1}^{n} \alpha_{j}=0$ one has

$$
\left\|\sum_{j=1}^{n} \alpha_{j} x_{j}\right\|=\sup \left\{\left|\sum_{j=1}^{n} \alpha_{j} f\left(x_{j}\right)\right|: f \in B_{L}\left(\left\{x_{1}, \ldots, x_{n}\right\}\right)\right\} .
$$

However, other properties of $\mathrm{CFL}(\mathbb{U})$ cannot be deduced from the above fact, and Holmes' theorem applies only to the unbounded Urysohn space. 
We end the paper with the following two questions. In both of them, $r$ is finite.

Question 1. The universality of an unbounded Urysohn space $\mathbb{U}$ and the results of Godefroy and Kalton [2] imply that the space CFL(U) is universal for separable Banach spaces (this was observed by Melleray [9]). These arguments do not work in the case of a bounded Urysohn space. Is the space $\mathcal{C}([0,1])$ isometrically or isomorphically embeddable in $\operatorname{CFL}\left(\mathbb{U}_{r}\right)$ ?

Question 2. Suppose that $(E,\|\cdot\|)$ is a Banach space such that $\mathbb{U}_{r} \subset E$ and $\|x-y\|=d(x, y)$ for all $x, y \in \mathbb{U}_{r}$. Does there exist a constant $c>0$ such that whenever $x_{1}, \ldots, x_{n}$ are points of $\mathbb{U}_{r}$ and $\alpha_{1}, \ldots, \alpha_{n}$ are real numbers with $\sum_{j=1} \alpha_{j}=0$, then

$$
\left\|\sum_{j=1}^{n} \alpha_{j} x_{j}\right\| \geq c \sup \left\{\left|\sum_{j=1}^{n} \alpha_{j} g\left(x_{j}\right)\right|: g \in B_{L}\left(\left\{x_{1}, \ldots, x_{n}\right\}\right)\right\} ?
$$

Does there exist a universal constant $c>0$ for which the above estimate holds (independently of the space $E$ )?

\section{References}

[1] S. Gao and A. S. Kechris, On the classification of Polish metric spaces up to isometry, Mem. Amer. Math. Soc. 161 (2003), no. 766.

[2] G. Godefroy and N. J. Kalton, Lipschitz-free Banach spaces, Studia Math. 159 (2003), 121-141.

[3] M. R. Holmes, The universal separable metric space of Urysohn and isometric embeddings thereof in Banach spaces, Fund. Math. 140 (1992), 199-223.

[4] - The Urysohn space embeds in Banach spaces in just one way, Topology Appl. 155 (2008), 1479-1482.

[5] G. E. Huhunaišvili, On a property of Urysohn's universal metric space, Dokl. Akad. Nauk SSSR (N.S.) 101 (1955), 332-333 (in Russian).

[6] M. Katětov, On universal metric spaces, in: General Topology and its Relations to Modern Analysis and Algebra VI (Prague, 1986), Z. Frolík (ed.), Heldermann, Berlin, 1988, 323-330.

[7] J. Melleray, On the geometry of Urysohn's universal metric space, Topology Appl. 154 (2007), 384-403.

[8] - Some geometric and dynamical properties of the Urysohn space, ibid. 155 (2008), 1531-1560.

[9] - Computing the complexity of the relation of isometry between separable Banach spaces, Math. Logic Quart. 53 (2007), 128-131.

[10] J. Melleray, F. V. Petrov and A. M. Vershik, Linearly rigid metric spaces and the embedding problem, Fund. Math. 199 (2008), 177-194.

[11] P. Niemiec, Integration and Lipschitz functions, Rend. Circ. Mat. Palermo 57 (2008), 391-399.

[12] —, Strengthened Stone-Weierstrass type theorem, to appear. 
[13] P. S. Urysohn, Sur un espace métrique universel, C. R. Acad. Sci. Paris 180 (1925), 803-806.

[14] —, Sur un espace métrique universel, Bull. Sci. Math. 51 (1927), 43-64, 74-96.

[15] N. Weaver, Lipschitz Algebras, World Sci., 1999.

Institute of Mathematics

Jagiellonian University

Łojasiewicza 6

30-348 Kraków, Poland

E-mail: piotr.niemiec@uj.edu.pl

Received March 25, 2008

Revised version November 28, 2008 